\title{
Parásitos intestinales en caninos y felinos con cuadros digestivos en Santiago, Chile. Consideraciones en Salud Pública
}

\author{
Javier López $\mathrm{D}^{1,2, a}$, Katia Abarca V3, \\ Patricio Paredes $M^{1, a, b}$, Elisa Inzunza $T^{2, c}$. \\ Intestinal parasites in dogs and cats \\ with gastrointestinal symptoms in \\ Santiago, Chile
}

Background: There is an increasing importance of pet-transmitted infections, some of those are considered emerging infections. Aim: To determine the species and frequency of intestinal parasites in pets with diarrhea (hemorrhagic gastroenteritis excluded). Material and methods: A descriptive retrospective study. Fecal samples from 972 dogs and 230 cats consulting in two veterinary practices in Santiago, between 1996 and 2003, were studied using Burrows' technique. Results: Protozoa were found in $64.8 \%$ of dogs and in $66.5 \%$ of cats; helminthes in $24 \%$ of dogs and $45.2 \%$ of cats. The species found in dogs were Blastocystis sp. in $36 \%$, Ameba sp. in 31\%, Giardia intestinalis in $22 \%$, Toxocara canis in $11 \%$, Chilomastix sp. in $10 \%$, Isospora sp. in 9\%, Trichuris vulpis in 9\%, Trichomonas sp. in 5\%, Sarcocystis sp. in 4\%, Dipylidium caninum in $2 \%$, Ancylostomideos in $2 \%$, Toxascaris leonina in $1 \%$, Physaloptera sp. in $1 \%$, Taenia sp. in $0.4 \%$. Species found in cats were Blastocystis sp. in 37\%, Ameba sp. in 30\%, G intestinalis in $19 \%$, Chilomastix sp. in $12 \%$, Isospora sp. in $12 \%$, Toxocara cati in $10 \%$, D caninum in $7 \%$, Sarcocystis sp. in $5 \%$, Trichomona sp. in 5\%, Toxoplasma gondii in $4 \%$, Taenia sp. in $2 \%$ and Physaloptera sp. in $1 \%$. Forty eight percent of parasites found in dogs and $49 \%$ found in cats have zoonotic potential. In dogs younger than six months Blastocystis sp., Ameba sp., G intestinalis, Chilomastix sp., Isospora sp. and T canis were significantly more common; the same was observed for Isospora in young cats. Approximately $60 \%$ of infected animals bore more than one parasite. Conclusions: A high rate of intestinal parasitism in pets with diarrhea was found; an important proportion of them have zoonotic potential (Rev Méd Chile 2006; 134: 193-200).

(Key w ords: Animals, domestic; Helminths; Parasites; Protozoa)

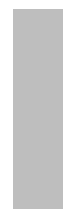

\footnotetext{
Recibido el 2 de marzo, 2005. Aceptado el 9 de agosto, 2005.

${ }^{1}$ Clínica Veterinaria Alcántara. ${ }^{2}$ Laboratorio de Parasitología Veterinaria, Clínica Alcántara.

${ }^{3}$ Centro de Investigaciones Médicas, Pontificia Universidad Católica de Chile.

aMédico Veterinario

bEstudiante de Medicina, Universidad de Chile

'Técnico en Parasitología
}

Correspondencia a: Javier López D. Clínica Veterinaria

Alcántara. Chacay 8835, La Florida, Santiago, Chile. Fono:

313 8061. Fax: 851 4769. E mail: javievet@entelchile.net 
$\mathrm{D}$ urante los últimos años han ido adquiriendo mayor relevancia las infecciones transmitidas por mascotas, algunas de las cuales se consideran infecciones emergentes. Sin duda, las mascotas más frecuentes en los hogares y que conviven más estrechamente con el ser humano son los perros (Canis familiaris) y los gatos (Felis catus). Estudios realizados en Santiago de Chile han establecido que $70 \%$ de los hogares posee una mascota (Abarca 2002, datos no publicados), con un promedio estimado de 0,78 perros y de 0,35 gatos por vivienda ${ }^{1}$.

El conocimiento de los agentes parasitarios intestinales de las mascotas que conviven más estrechamente con el hombre tiene implicancias tanto en medicina veterinaria como en salud humana, ya que varios agentes tienen la potencialidad de transmitirse del animal al humano y viceversa.

En Chile se han encontrado en perros, por exámenes coproparasitarios, infecciones que oscilan entre 27 y $50 \%^{2,3}$ y en gatos, a través de estudios post-mortem, cifras aún mayores (84$99 \%)^{4,5}$. Hasta la fecha, se han reportado en nuestro país, en perros, gatos o ambos, las siguientes especies de protozoos y helmintos: Giardia intestinalis, Isospora sp., Sarcocystis sp., Cryptosporidium sp., Toxoplasma gondii, Tripanosoma cruzi, Ollulanus tricuspis, Toxocara canis, Toxocara cati, Toxascaris leonina, Strongyloides stercoralis, Filaroides osleri, Trichuris vulpis, Ancylostomideos (Ancylostoma caninum y Uncinaria stenocephala), Capillaria aerophila, Capillaria plica, Aelurostrongylus abstrusus, Dipetalomena reconditum, Trichinella spiralis, Dipylidium caninum, Echinococcus granulossus, Diphyllobotrium latum, Spirometra mansoni, Mesocestoides lineatus, Taenia hydatigena, Taenia multiceps, Taenia serialis, Taenia pisiformis, Taenia taeniaeformis, Phagicola sp., Echinochasmus sp. ${ }^{2-10}$.

Estos estudios generalmente han usado técnicas de flotación para la observación de las deposiciones $^{2,8}$. En humanos y en animales se ha demostrado que técnicas de centrifugación y sedimentación, como Telemann y Burrows, facilitan la observación de protozoos ${ }^{4,11,12}$. Esto lo corroboran algunos estudios que, usando técnica de Telemann, encuentran protozoos no observados previamente, como $G$ intestinalis ${ }^{4}$. Se ha demostrado, además, que la técnica de Burrows presenta mejor sensibilidad en relación a Telemann para formas trofozoíticas, especialmente de amebas y giardias ${ }^{13}$. No existen en Chile estudios de parasitismo en animales domésticos que hayan empleado la técnica de Burrows, y son escasos los estudios realizados en animales con cuadros digestivos $^{2}$.

Los objetivos del presente estudio fueron determinar frecuencia y tipo de parásitos intestinales en caninos y felinos consultantes por cuadros digestivos en una clínica veterinaria de Santiago, evaluar si existen diferencias en la frecuencia de los distintos parásitos en cachorros con relación a los animales mayores, estimar la proporción de estas infecciones que tendrían potencial zoonótico y discutir las implicancias en salud pública de los hallazgos.

\section{Material y MÉTodo}

La población estuvo constituida por 972 perros y 230 gatos, consultantes por deposiciones alteradas o diarrea franca, en una clínica veterinaria del área sur de Santiago, entre junio de 1996 y diciembre de 2003. Se excluyeron cuadros de gastroenteritis hemorrágica aguda.

Los dueños de las mascotas recolectaron 3 muestras de deposiciones frescas, día por medio, en frascos con PAF (fenol, alcohol y formaldehído) como fijador.

Las muestras fueron procesadas en el Laboratorio de la Clínica Veterinaria Alcántara por un técnico con amplia experiencia en parasitología, mediante procedimiento adaptado de la técnica de Burrows ${ }^{11}$. Brevemente, las muestras de cada animal se mezclaron en un recipiente y se diluyeron con suero fisiológico. De este material, se extrajo una muestra para observación directa con tionina y otra con colorante MIF (merthiolate, yodo y formaldehído) con aumento 10X y 40X. Cuando se encontraron elementos sugerentes de amebas, se observó con aumento 100X y aceite de inmersión. La emulsión restante se dividió en dos, una para observación de trofozoítos y otra para formas quísticas. Para las formas trofozoíticas, se centrifugó la muestra durante $4 \mathrm{~min}$ a $1.500 \mathrm{rpm}$, se eliminó el sobrenadante y se lavó el sedimento con suero fisiológico. Se repitió este proceso de lavado 2 ó 3 veces. El sedimento resultante se 
observó con tinciones tionina y MIF. Para las formas quísticas, se realizó un tamizaje de las deposiciones con rejilla de cobre fosforado de 37 $\mathrm{mm}$. A $15 \mathrm{~mL}$ de deposiciones tamizadas se agregó $1 \mathrm{~mL}$ de éter, se agitó, se centrifugó por 4 min a 1.500-1.800 rpm, se eliminó el sobrenadante y se observó el sedimento con tinciones tionina y MIF.

Debido a que en ciertos casos el diagnóstico de certeza de especie requiere de estudios moleculares o microscopia electrónica, las distintas especies de los géneros Blastocystis, Chylomastix, Trichomonas y Sarcocystis se presentan bajo la nomenclatura sp. Bajo el término Isospora sp., se incluyen las especies I. canis, I. ohioensis, I. felis e I. rivolta.

Bajo la nomenclatura Ancylostomideos se incluyen Uncinaria stenocephala y Ancylostoma caninum. El diagnóstico de Physaloptera se basó en la morfología de huevos, no se recolectaron ejemplares adultos para un diagnóstico de especie.

Se registró en una base de datos Access: especie del animal, edad y parásitos encontrados. En aquellos casos en que se contaba con el antecedente de edad del animal, se comparó la frecuencia de protozoos intestinales entre menores y mayores de 6 meses de edad, mediante test de Fisher.

\section{RESULTADOS}

Se encontró algún protozoo en 630 de los perros $(64,8 \%)$ y en 153 de los gatos $(66,5 \%)$. Las especies encontradas se indican en la Tabla 1. En la comparación por grupos etarios, se encontró significativa mayor frecuencia de Blastocystis, Ameba, Giardia, Chilomastix e Isospora en perros menores de 6 meses y de Isospora en gatos menores de 6 meses (Tabla 2).

Se encontró algún helminto en 234 de las muestras de perros (24\%), y en 104 de gatos $(45,2 \%)$. Las especies encontradas se indican en la Tabla 3. Al analizar la frecuencia de helmintos por edad del animal, se encontró frecuencia significativamente mayor de $\mathrm{T}$. canis en perros menores de 6 meses $(p<0,0001)$. Se observó una tendencia a mayor frecuencia de T. catis y D. caninum en cachorros de gatos, pero sin alcanzar significancia estadística.

De las 686 muestras de perros positivas para protozoos, helmintos o ambos, en 431 (63\%) se encontró más de un parásito. En 36\% se encontraron dos, en $17 \%$, tres, y en $9 \%$, cuatro o más parásitos. De las 159 muestras de gatos positivas, en 92 (58\%) se encontró más de un parásito. En $29 \%$ se encontraron dos, en $20 \%$, tres, y en $9 \%$, cuatro o más parásitos.

Tabla 1. Frecuencia de protozoos encontrados en deposiciones de perros y gatos

\begin{tabular}{|lrrrr|}
\hline Géneros/ especies & \multicolumn{2}{c}{ Perros (n=972) } & \multicolumn{2}{c|}{ Gatos (n=230) } \\
& $\mathrm{N}$ & $\%$ & $\mathrm{~N}$ & $\%$ \\
\hline Blastocystis sp & 351 & 36,1 & 86 & 37,4 \\
Endolimax nana & 334 & 34,3 & 74 & 32,2 \\
Entamoeba coli & 322 & 33,1 & 73 & 31,7 \\
Entamoeba histolytica & 316 & 32,5 & 70 & 30,4 \\
Giardia intestinalis & 211 & 21,7 & 44 & 19,1 \\
Chilomastix sp & 92 & 9,5 & 28 & 12,2 \\
Isospora sp & 89 & 9,2 & 28 & 4,8 \\
Trichomonas sp & 46 & 4,7 & 11 & 5,2 \\
Sarcocystis sp & 35 & 3,6 & 12 & 4,3 \\
Toxoplasma gondii & - & - & 10 & \\
\hline
\end{tabular}


Tabla 2. Frecuencia de protozoos por grupos etarios, en perros y gatos

\begin{tabular}{|c|c|c|c|c|c|c|}
\hline \multirow[t]{2}{*}{ Géneros/especies } & \multicolumn{3}{|c|}{ Perros } & \multicolumn{3}{|c|}{ Gatos } \\
\hline & $<6$ meses & $>6$ meses & $\mathrm{p}$ & $<6$ meses & $>6$ meses & $\mathrm{p}$ \\
\hline Blastocystis sp & $143 / 190$ & $47 / 190$ & $<0,0001$ & $18 / 32$ & $14 / 32$ & NS \\
\hline Ameba sp & $118 / 176$ & $58 / 176$ & 0,0038 & $11 / 22$ & $11 / 22$ & NS \\
\hline Giardia intestinalis & $99 / 119$ & $20 / 119$ & $<0,0001$ & $12 / 19$ & $7 / 19$ & NS \\
\hline Chilomastix sp & $47 / 61$ & $14 / 61$ & 0,0014 & $6 / 14$ & $8 / 14$ & NS \\
\hline Isospora sp & $40 / 46$ & $6 / 46$ & $<0,0001$ & $10 / 12$ & $2 / 12$ & 0,0242 \\
\hline Trichomonas sp & $21 / 31$ & $10 / 31$ & NS & $3 / 5$ & $2 / 5$ & NS \\
\hline Sarcocystis sp & $10 / 20$ & $10 / 20$ & NS & $4 / 7$ & $3 / 7$ & NS \\
\hline Toxoplasma gondii & - & - & - & $2 / 3$ & $1 / 3$ & NS \\
\hline
\end{tabular}

Tabla 3. Frecuencia de helmintos encontrados en deposiciones de perros y gatos

\begin{tabular}{|lrrrr|}
\hline Géneros/especies & \multicolumn{2}{c}{ Perros $(\mathrm{n}=972)$} & \multicolumn{2}{c|}{ Gatos $(\mathrm{n}=230)$} \\
& $\mathrm{n}$ & $\%$ & - & $\%$ \\
\hline Toxocara canis & 108 & 11,1 & 23 & - \\
Toxocara cati & - & - & - & - \\
Trichuris vulpis & 86 & 8,9 & 16 & 6,9 \\
Dypylidium caninum & 21 & 2,2 & 0 & 0 \\
Ancylostomoideos & 17 & 1,8 & 0 & 0 \\
Toxascaris leonina & 14 & 1,4 & 2 & 0,9 \\
Physaloptera sp & 12 & 1,2 & 4 & 1,7 \\
Taenia sp & 4 & 0,4 & & -9 \\
\hline
\end{tabular}

Respecto a la potencial transmisión de los parásitos intestinales de las mascotas al hombre, $371 / 1.390(26,7 \%)$ de los agentes encontrados en perros y $95 / 333(28,5 \%)$ en gatos tendrían potencial zoonótico (G. intestinalis, T. gondi, T. canis, Ancylostomideos, D. caninum y T. cati). Si se agrega E. histolytica, considerada una antropozoonosis, la cifra se eleva a 48,4\% (673/1.390) en perros y a $49,5 \%(165 / 333)$ en gatos.

\section{DISCUSIÓN}

Es destacable el elevado porcentaje de parasitismo intestinal encontrado en mascotas con cuadros digestivos: protozoos en $64,8 \%$ y helmintos en $24 \%$ de los perros, protozoos en $66,5 \%$ y helmintos en $45,2 \%$ de los gatos. En el caso de los perros, estas cifras son mayores a las reportadas en estudios chilenos previos, que indicaban una frecuencia global de parásitos entre 27 y 50\%2,4. En gatos, se habían reportado tasas más altas (frecuencia global de 84-99\%)6,7, pero en animales post mortem, por lo que no son comparables. Coincidentemente con lo descrito por otros autores, se encontró un mayor índice de algunas parasitosis en cachorros que en animales adultos 2,14 .

Esta elevada tasa de parásitos intestinales encontrada en mascotas, especialmente elevada 
en el caso de los protozoos, parece indicar que existen importantes fallas en las medidas preventivas de estas infecciones en las mascotas en nuestro medio, tanto en la prevención individual (uso de antiparasitarios) como colectiva (reducción de la contaminación ambiental). Respecto a profilaxis individual, las medidas indicadas habitualmente por los veterinarios incluyen el uso de medicamentos antiparasitarios, medicamentos de acción principalmente anti-helmíntica, con escaso potencial de erradicación de protozoos. La cobertura real de estas medidas depende principalmente de la atención veterinaria de las mascotas y ésta, del nivel socioeconómico (NSE) de las familias y del tipo de mascotas. Un estudio chileno encontró que $92 \%$ de los perros de NSE alto y $42 \%$ de NSE medio-bajo están en control veterinario, cifras que bajan a $71 \%$ y $21 \%$, respectivamente en los gatos (Abarca 2002, datos no publicados). Así, los gatos tienen un menor acceso a control veterinario $\mathrm{y}$, por tanto, a desparasitación rutinaria. En lo que se refiere al ámbito colectivo, distintos estudios nacionales y latinoamericanos han demostrado un elevado grado de contaminación por parásitos en lugares públicos como parques, plazas de juegos y playas ${ }^{15-17}$. El elevado número de perros vagabundos, la ausencia de tenencia responsable de mascotas y de una legislación adecuada en la eliminación de excretas en sitios públicos, son factores que explicarían esta situación.

Entre los protozoos encontrados, las mayores frecuencias corresponden a Blastocystis sp., Amebas sp. y $\mathrm{G}$ intestinalis. Coincidentemente, estudios realizados en Chile y otros países de Latinoamérica han mostrado que especies pertenecientes a estos tres géneros son las más frecuentemente encontradas en humanos, tanto asintomáticos como con cuadros diarreicos ${ }^{17,18}$.

Si bien Blastocystis ha sido descrito en perros y gatos $^{19}$, su potencial zoonótico no ha sido aclarado. Un estudio encontró significativa mayor frecuencia de infección por este agente en personas que trabajan con animales ${ }^{20}$. Sin embargo, otros autores no encuentran asociación entre portación de Blastocystis y presencia de animales domésticos en el hogar, pero sí con el consumo de agua $\sin$ hervir $^{21}$. Una limitación de nuestro estudio es que, al no incluir microscopia electrónica ni estudios moleculares de Blastocystis, no se pudo llegar a un diagnóstico certero de especie. Sería interesante realizar estudios que permitan identificar la especie de Blastocystis presente en mascotas, de forma de aclarar el posible rol zoonótico de este agente en Chile, sobre todo considerando que es el principal agente encontrado en este estudio.

En el caso de las amebas, el reservorio natural lo constituye la especie humana, perros y gatos serían huéspedes accidentales, constituyendo un ejemplo de antropozoonosis ${ }^{22}$. La frecuencia encontrada de amebas en este estudio fue elevada (un tercio de las mascotas estudiadas), y mucho mayor a la encontrada en otros países. Lo anterior podría reflejar un alto grado de parasitación en personas 0 en el ambiente.

Recientemente se ha reconocido el rol zoonótico de Giardia. Se ha demostrado que giardias de hombre y animales domésticos son morfológica y genéticamente similares, existiendo varias observaciones que sugieren la posibilidad de infecciones cruzadas ${ }^{23-25}$. Esta similitud en las especies que afectan a humanos, animales domésticos y otros mamíferos, llevó a agruparlas bajo la nomenclatura de G. intestinalis, encontrándose en otros grupos las especies que afectan a aves, roedores, reptiles y anfibios ${ }^{20}$. La frecuencia de G. intestinalis en este estudio fue notablemente mayor que en estudios previos. Esto probablemente se debe a que se trata de animales con cuadros diarreicos. G. intestinalis parece ser entonces un parásito relevante tanto por su frecuencia (presente en $1 / 6$ y $1 / 5$ de los perros y gatos con cuadros diarreicos, respectivamente) como por su comprobado rol en infecciones zoonóticas.

Según el conocimiento de los autores, se describen en este estudio, por primera vez en mascotas chilenas, cinco agentes parasitarios, pertenecientes a cuatro géneros de protozoos: amebas, Chilomastix, Trichomonas y Blastocystis y un helminto: Physaloptera. Estos protozoos, con excepción de Chilomastix, han sido encontrados en perros o gatos en otros países ${ }^{19,22,26,27}$. La especie Ch. mesnili ha sido descrita en humanos, primates y cerdos; otras especies se han encontrado en animales como equinos, caprinos, aves, cobayos y conejos $^{22}$, sin que los autores hayan encontrado reportes previos de su presencia en perros ni gatos. Debe considerarse que el presente estudio se basa en observación de microscopia de luz, que no 
permite un diagnóstico de certeza de la especie, por lo que son necesarios estudios que determinen la especie de Chilomastix presente en mascotas. La ausencia de descripción previa de estos protozoos en perros y gatos en otros estudios chilenos podría explicarse por el uso de técnicas de flotación en ellos, de menor sensibilidad para estos agentes que la técnica de sedimentación ${ }^{4,11,12}$.

Physaloptera ha sido descrita en forma aislada en perros y gatos fuera de Chile, pero no existían estudios de su frecuencia en estos animales. Este agente no corresponde a una zoonosis, pues la especie infectante de humanos corresponde a Physaloptera caucasica, la cual ha sido encontrada en humanos en Chile $^{28}$.

El rol de algunos parásitos intestinales de perros y gatos como agentes de infecciones zoonóticas ha sido claramente establecido desde hace varias décadas para especies como T. gondii, T. canis, Ancylostomideos, D. caninum, E. granulossus y T cati.

T. gondii es uno de los agentes más importantes de infecciones zoonóticas en humanos. A pesar que el reservorio natural de esta infección es el gato, diversos estudios han demostrado que el mayor riesgo de adquisición lo constituye la ingesta de carne mal cocida y no el contacto directo con los gatos ${ }^{29}$.

Coincidentemente con otros estudios, se encontró una elevada frecuencia de parasitación de las mascotas por agentes productores del síndrome de larva migrante cutánea y visceral, zoonosis de enorme importancia en medicina humana, ampliamente documentada en nuestro país ${ }^{30,31}$. Además de su rol establecido en los síndromes de larvas migrantes, existen evidencias que $\mathrm{T}$. canis podría tener un rol en cuadros de urticaria crónica $^{32}$ y Ancylostoma caninum en enteritis eosinofílica en humanos ${ }^{33}$. Si bien la transmisión de $T$. canis al humano, tradicionalmente, se ha

\section{REFERENCIAS}

1. IbarRa L, Morales MA, AcuÑa P. Aspectos demográficos de la población de perros y gatos en la ciudad de Santiago, Chile. Avs Cs Vet 2003; 18: 1320. atribuido a geofagia, recientes estudios han encontrado huevos infectantes en el pelaje de perros, sugiriendo la posibilidad de transmisión por contacto directo ${ }^{34}$. En Chile, se ha reportado infección humana por D. caninum, afectando generalmente a niños pequeños ${ }^{35,36}$.

Hasta la fecha se acepta que algunos parásitos son especies específicas, por lo que no tienen potencial zoonótico, como los pertenecientes a los géneros Isospora, Physaloptera, Trichomonas y las especies de Taenias encontradas en gatos y perros, diferentes de E. granulossus. La menor frecuencia de Isospora encontrada en gatos en relación a estudios previos podría deberse a no haber utilizado técnica de flotación en este estudio, la que ha demostrado mayor rendimiento en la detección de este agente en particular ${ }^{4}$.

En otras especies parasitarias, el rol zoonótico está en discusión o es desconocido. Por ejemplo, existe cierta evidencia, aún no ampliamente aceptada que $\mathrm{T}$. vulpis podría transmitirse de mascotas al humano ${ }^{24}$. La información referente a T. leonina como agente zoonótico es controversial, siendo señalado por algunos autores como uno de los agentes causales de síndromes de larvas migrantes $^{37}$ y por otros no ${ }^{24}$. En el caso de Sarcocystis, la transmisión a humanos ocurre mediante ingesta de carne de vacuno contaminada, pero no se ha descrito la transmisión desde mascotas ${ }^{24}$.

El importante porcentaje de los parásitos encontrados en perros y gatos, que tienen potencial zoonótico (aproximadamente la mitad), refuerza aún más la necesidad de instaurar efectivas medidas de prevención no sólo de las parasitosis en los animales sino de la transmisión hacia humanos. Para optimizar esta necesaria prevención se requiere de mayor conocimiento de las infecciones con potencial zoonótico en nuestro medio y de una mayor integración entre médicos veterinarios y de salud humana.

2. Alcaíno H, Tagle I. Estudio sobre enteroparasitosis del perro en Santiago. Bol Chil Parasitol 1970; 25: 5-9.

3. Gorman T, Yáñez V, Alcaíno H. Coccidias intestinales en caninos de la comuna de San Miguel, Región Metropolitana, Chile. Avs Cs Vet 1989; 4: 57-62. 
4. Torres P, Hott A, Bohenwald H. Protozoos, helmintos y artrópodos en gatos de la ciudad de Valdivia y su importancia para el hombre. Archivos Med Veterinaria 1972; 4; 20-9.

5. Torres $\mathrm{P}$, Ramos $\mathrm{R}$, Carrasco L, Neumann $\mathrm{M}$, Franjola R, NavarRete N et al. Protozoos, helmintos y artrópodos parásitos del perro doméstico de la ciudad de Valdivia, Chile. Bol Chil Parasitol 1974; 29: 18-23.

6. Alcaíno H, Gorman T, Larenas I. Fauna endoparasitaria del gato doméstico proveniente de zona urbana marginal. Parasitol al Día 1992; 16: 139-42.

7. Franjola R, Matzner N. Prevalencia de enteroprotozoos en gatos domésticos en la ciudad de Valdivia. Zbl Vet Med B 1982; 29: 397.

8. Torres A. Sarcocystis sp en el diafragma de un gato doméstico. Bol Chil Parasitol 1996; 51: 30-2.

9. Neghme A, Rivera G, Alvarez M. Algunas zoonosis parasitarias en perros vagos de la ciudad de Santiago. Bol Chil Parasitol 1955; 4: 73-5.

10. Alcaíno H, Gorman T. Parásitos de los animales domésticos en Chile. Parasitol al Día 1999; 23: 33-41.

11. Burrows RB. A new fixative and techniques for the diagnosis of intestinal parasites. Amer J Clin Pathol 1967; 48: 342-6.

12. Lorca M, Astorga B. Técnicas de laboratorio. En: Atías, Neghme, ed. Parasitología Clínica. Editorial Mediterráneo 1979; 501-24.

13. Galdames M, Zuloaga M, Jarpa A. Diagnóstico coproparasitario mediante los métodos de Telemann modificado y de Burrows. Bol Chil Parasitol 1984; 39: 10-2.

14. Bartmann A, Pacheco FA. Frequency of Giardia lamblia in dogs attended by veterinary clinics in Porto Alegre City, RS, Brazil. Cienc Rural 2004; 34: 1093-6.

15. Castillo D, Paredes C, Zañartu C, Castilo G, Mercado R, Muñoz V et al. Contaminación ambiental por Toxocara sp. en algunas plazas y parques públicos de Santiago de Chile, 1999. Bol Chil Parasitol 2000; 55: 86-91.

16. Mercado R, Ueta MT, CastiLo D, Muñoz V, SCHENONE H. Exposure to larva migrans syndromes in squares and public parks of cities in Chile. Rev Saúde Pública 2004; 38: 729-31.

17. Andresiuk MV, Denegri GM, Esardella NH, HoLman P. Encuesta coproparasitológica canina realizada en plazas públicas de la ciudad de Mar del Plata, Buenos Aires, Argentina. Parasitol Latinoam 2003; 58: 17-22.
18. Mercado R, CastiLo D, Muñoz V, Sandoval L, Jercic MI, GIL JC ET AL. Infecciones por protozoos y helmintos intestinales en pre-escolares y escolares de la Comuna de Colina, Santiago, Chile. Parasitol Latinoam 2003; 58: 173-6.

19. Duda A, Stenzel DJ, Borcham PF. Detection of Blastocystis sp. in domestic dogs and cats. Vet Parasitol 1998; 76: 9-17.

20. Rajah Salim H, Suresh KG, Vellayan S, Mak JW, Khaiul Anuar I, Vennila GD et al. Blastocystis in animal handlers. Parasitol Res 1999; 85: 1032-3.

21. Barahona L, Maguña C, Náquira C, Terashima A, Тецо R. Sintomatología y factores epidemiológicos asociados al parasitismo por Blastocystis hominis. Parasitol Latinoam 2002; 57: 96-102.

22. SoulsBy EJL. Parasitología y Enfermedades Parasitarias en los Animales Domésticos. Editorial Interamericana 1987, 822 págs.

23. Meloni BP, Lymbery AJ, Thompson RC. Genetic characterization of isolates of Giardia duodenalis by enzyme electrophoresis: implications for reproductive biology, population structure, taxonomy, and epidemiology. J Parasitol 1995; 81: 368-83.

24. Acha P, SZyFres B. Zoonosis y enfermedades transmisibles comunes al hombre y a los animales. Volumen III Parasitosis. Tercera edición. Editorial OPS, 2003, 403 págs.

25. Traub RJ, Monis PT, Robertson I, Irwin P, Mencke N, Thompson RC. Epidemiological and molecular evidence supports the zoonotic transmission of Giardia among humans and dogs living in the same community. Parasitology 2004; 128: 253-62.

26. Gookin JL, Breitschwerdt EB, LeVy MG, Pager RB, BENRUd JG. Diarrhea associated with trichomonosis in cats. J Am Vet Med Assoc 1999; 215: 1450-4.

27. Zárate JJ, Craig T, Avalos R, Guzmán, M, Dávalos G, RAmirez R. Gastritis verminosa por Physaloptera praeputialis en el gato. Vet Méx 1991; XXII; 2, 185-90.

28. Apt W, Sapunar J, Doren G, Rojo M. Physaloptera caucasica. Primeros casos humanos en Chile. Bol Chil Parasitol 1965; 20: 111.

29. Cook AJC, Gilbert RE, Buffolano W, Zufferey J, Petersen E, Jenum PA et al. Sources of Toxoplasma infection in pregnant women: European multicentre case-control study. BMJ 2000; 321: 142-7.

30. Herskovic P, Astorga B. Toxocariasis humana en Chile. Rev Méd Chile 1985; 113: 18-31. 
31. Triviño $\mathrm{X}$, Bedregal $\mathrm{P}$, Torres $\mathrm{M}$, Canales $\mathrm{M}$, Alvarado C, Hernández R. Toxocarosis en Chile: Serie clínica en un centro de Pediatría Ambulatoria. Parasitol al Día 1999; 23: 113-8.

32. Wolfrom E, Chene G, Lejoly-Bosseau H, Beylot C, Genuiaux M, TaieB A. Chronic urticaria and Toxocara canis infection. A case-control study. Ann Dermatol Venereol 1996; 123: 240-6.

33. Croese J, Loukas A, Opdebeeck J, Fairley S, Prociv P. Human enteric infection with canine hookworms. Ann Intern Med 1994; 120: 369-74.

34. Wolfe A, Wright IP. Human toxocariasis and direct contact with dogs. Vet Rec 2003; 152: 419-22.
35. Belmar R. Dypilidium caninum en niños. Comunicación de 13 casos y tratamiento con un derivado de la salicilamida. Bol Chil Parasitol 1963; 28: 63-7.

36. Reyes H, Doren G, Inzunza E. Teniasis humana. Frecuencia actual de la infección por diferentes especies en Santiago de Chile. Bol Chil Parasitol 1972; 27: 23-9.

37. Noemí I, Rugiero E. Larvas migrantes. En: Atías, ed. Parasitología Médica. Santiago: Editorial Mediterráneo, 1998; 332-7. 